simple to Stokes and Thomson in 1852 , and to Stewart and Kirchhoff a few years later.

I wish to consider briefly, what are these new and puzzling complications of the solar problem; and whether we may not still preserve our belief in the existence of essentially different elementary atoms, which is the basis of tine beautiful Vortex Theory. For it seems that to hazard (however naturally) such a step as is involved in assumed dissociation of the (so-called) elements, before we make certain that no less serious hypothesis will account for the observed facts, is contrary to the spirit of Newton's Regule Philosophandi.

The most prominent of these complications seem to be-

(1). The variations of the relative brightness, width, \&c., of the lines in the spectrum of a particular substance, in dependence on the source and circumstances of its incandescence.

(2). The so-called "long" and "short" lines. (These, as will be seen, are probably a case of (1).)

(3). The fact that, in the spectra of sun-spots, some lines supposed to be due to a particular element indicate rapid motion of the glowing gas; while others, supposed due to the same element, give no such indication.

(4). The (at least apparent) coincidence of lines in the spectra of two or more elementary substances.

To these may be added:-

(5). The remarkable peculiarities of star-spectra; especially the paucity, and the breadth, of the lines in the spectra of white stars.

As regards (1), let us consider a sounding body with a large number of different modes of vibration, exposed to impacts either periodic or at least with an average period. The relative intensities of the various notes which it can give will obviously depend upon the period of the impacts. Now this is precisely the case of a particle (I use the word to avoid misconception) of a glowing gas. The average number of blows it receives will depend on $(a)$ the number of particles per cubic inch (and also upon whether thure be another gas present or no, a point of very great importance) and (b) the te aperature, which is directly connected with the velocity of the particles.

Change the density, the temperature, the admixture with foreign substances, or any two, or all, of these; and the average period of the battering to which a particle is subjected may be so altered as to elicit from it in any required ratios of relative intensity the various simple rays it can give out.

It will readily be seen that this may account for all of the phenomena of classes ( 1 ) and (2) above.

(3) may be accounted for in many ways. I mention only one, as my object is merely to show that we are not yet compelled to accept dissociation of so-called elements even in its mildest form. Other modes of escape, though not quite so simple, present themselves.

What is seen in a sun-spot is the integral, as it were, of all that is taking place (as regards both radiation and absorption) in many thousand mile; of solar atmosphere, containing the same su'stance under the most varied conditions. That portions in which certain lines of that substance are prominent over other may be at rest relatively to the observer along the line of sight; while others, in which (from different density, temperature, or admixture, as ab ove explained) other lines are specially prominent, may bave large relative velositie; is certain. This would at once account for these singular observations.

As to (5) we must remeinber that in a star spectrum we have, as it were, a triple integral. For we not only interrate through the depth of the atmosphere, but also over the whole surface of the star ; spots, hurricanes, and rotation of the whole, included. This is equivalent to the superposition of innumerable separate spectra, no two of which may have any one individual line in the same place or of the same breadth, \&c. Feeble lines may, in fact, entirely disappear under such treatment.

(4) If not due to want of dispersive power in the apparatus, this may be legitimately attributed to inevitable impurities. It is only in "tall talk" (or in advertisements) that any human preparation, elementary or not, can be spoken of as "chemisch rein." And we all know how faint a trace of impurity can be detected by the help of the spectroscope.

Even in the last resort, I see nothing to hinder the existence of exactly equal vibration-periods in two perfectly distinct vortexatoms :-though their occurrence i; extremely improbable.

If we could get an absolutely transparent gas; one, therefore, which could give no radiation under any circumstances; the study of the behaviour of a given quantity of hydrogen mixed with dif- ferent proportions of it in a vessel of given size, and subjected always to the same conditions of incandescence, would give us invaluable information.

G. H.

\section{Replacing Flakes on Palæolithic Implements}

THIs wonderful feat was first performed by my friend $\mathrm{Mr}$. F. C. J. Spurrell of Dartford. On first thoughts the thing seems utterly impossible, and it is obvious that no flake can possibly be replaced upon an implement unless one lights on the exact spot where the instrument was made, and finds both imple ment and flakes in position. Mr. Spurrell so found his material. During the present summer I have discovered another and similar Palæolithic floor, far removed from Mr. Spurrell's, and where implements and flakes are exposed in a stratum perfectly undisurbed since they were gently covered up in Palæolithic times with fine sand containing the shells of such freshwater molluses as Unio, Cyrenia, and Bythinia. For obvious reasons-the chief one of which is that my work would be totally stopped if I mentioned the locality-I will content myself with stating that the position is nearly a mile from any river, and the floor is $4^{r}$ feet above tha level of the nearest stream; above the floor is a thick deposit of fine stratified sand, and above that loam. On this Palæolithic floor I have found several implement; and a large number of flakes, and on one of the finest implements, an example 6 inches long, $3 \frac{1}{8}$ inches wide, and weighing $\mathrm{I} \frac{1}{4} \mathrm{lb}$ I have been able to replace two flakes, one $2 \frac{1}{4}$ inches $13 n g$, the other 2 inches in exact position; the flakes slightly overlap each other on the implement, and bo:h have been stiuck from the edge of the ionplement at right angles across its face. The implement and flakes were close toxether, and with them found a hammer-stone of fliot with a distinctly battered and abraded edge. Mr. Spurrell replaced many flakes round his implenent, but the implement itself was a spoilt and poor example. My implement, on the contrary, is an unusually fine one, large, heavy, and perfect. Both the implement and flakes show a little of the original grey crust of the flint from which the instrument was made, and this peculiar grey colour led me to attempt the replacennent of the flakes with the above-mentioned successful result. One flake has a slirhtly uneven ed gein some instances considered a proof of $u$ :e-the sec ond flake is quite sharp. I shall exhibit this implement, with other implements, flake; \&c., fro n the same place, at an early meetiag of the Anthropological Institute. Worthingron G. Smiti I25, Grosveno: Roał, Highbury, N.

\section{Integrating Anemometer}

Perhaps the following brief desciption of the interrator devised by me will suffice to establish its near kinship with $\mathrm{Mr}$. Wils on's (NATURE, vol. xxiv. pp. 467 and 557):-A roller with a spherical edge is made to revolve with a velocity proportional to that of the wind as recorded on an anemogram. This roller presses on a plane table carried by two mutually perpendicular pairs of rails in planes parallel to that of the table. The lowest of the pairs of rails is supported by a frame carried on the extremity of a vertical shaft. The point of contact of the roller with the table lies in the prolongation of the axis of the shaft. The table can rotate with the shaft, but not independently. By a siople arrangement the shaft, and consequently the table, are caused to take up positions corresponding from moment to monent with the direction of the wind record on the anemogram. A style concentric with the shaft presses lightly against a compound sheet of tracing and carbonised paper attached to the under side of the table. Arrangements are also made for obtaining the sum of the movements of the table toward each of the four cardinal points. If the roller be moved with a velocity proportional to that of the wind, whether directly by a cup-anemometer or by a mechanical translation of the trace as given by stich an instrument, while the table simultaneously assumes orientations corresponding to the direction of movement of the air, the line drawn by the style will be a miniature copy of the path of an imaginary particle animated by the movements actually belonging to the masses of air which successively affect the anemometer at the given station during the selected period, rigorously in accordance with the principle known as Lambert's. But in order that the trace drawn as described should correctly represent the actual movements of the air, it is evident that the whole mass of the atmosphere must be supposed to move "parallel to itself," i.e. in such a manner that the straight 